\title{
Glioblastoma Recurrence Versus Treatment Effect in a Pathology- Documented Series
}

\author{
Benjamin T. Himes (1D, Andrea L. Arnett, Kenneth W. Merrell, Marcus J. Gates, \\ Adip G. Bhargav, Aditya Raghunathan, Desmond A. Brown, Terry C. Burns, \\ Ian F. Parney
}

\begin{abstract}
Objective: Patients diagnosed with glioblastoma (GBM) are treated with surgery followed by fractionated radiotherapy with concurrent and adjuvant temozolomide. Patients are monitored with serial magnetic resonance imaging (MRI). However, treatment-related changes frequently mimic disease progression. We reviewed a series of patients undergoing surgery for presumed first-recurrence GBM, where pathology reports were available for tissue diagnosis, in order to better understand factors associated with a diagnosis of treatment-related changes on final pathology. Methods: Patient records at a single institution between 2005 and 2015 were retrospectively reviewed. Pathology reports were reviewed to determine diagnosis of recurrent GBM or treatment effect. Survival analysis was performed interrogating overall survival (OS) and progression-free survival (PFS). Correlation with radiation treatment plans was also examined. Results: One-hundred-twenty-three patients were identified. One-hundred-sixteen patients (94\%) underwent resection and seven underwent biopsy. Treatment-related changes were reported in 20 cases (16\%). These patients had longer median OS and PFS from the time of recurrence than patients with true disease progression. However, there was no significant difference in OS from the time of initial diagnosis. Treatment effect was associated with surgery within 90 days of completing radiation. In patients receiving radiation at our institution $(n=53)$, larger radiation target volume and a higher maximum dose were associated with treatment effect. Conclusion: Treatment effect was associated with surgery nearer to completion of radiation, a larger radiation target volume, and a higher maximum point dose. Treatment effect was associated with longer PFS and OS from the time of recurrence, but not from the time of initial diagnosis.
\end{abstract}

RÉSUMÉ : Comparaison documentée entre des cas de récidive de glioblastome et les effets liés à des traitements. Objectif : Les patients à qui l'on a diagnostiqué un glioblastome (GBM) peuvent être traités par une chirurgie suivie, de façon simultanée, de séances fractionnées de radiothérapie et d'un traitement adjuvant au témozolomide. Le suivi des patients est ensuite effectué au moyen d'examens d'IRM. Cela dit, les changements à leur état de santé pouvant être liés à ces traitements se confondent fréquemment avec la progression de la maladie elle-même. Nous avons ainsi passé en revue les cas de patients ayant subi une intervention chirurgicale en lien avec une première récurrence présumée de GBM. Leurs rapports pathologiques étant disponibles, il a été possible d'effectuer un diagnostic tissulaire afin de mieux comprendre, dans le cas d'une pathologie finale, les facteurs associés à un diagnostic de changements à leur état de santé en lien avec un traitement. Méthodes : Nous avons passé en revue de façon rétrospective les dossiers de patients traités dans un seul établissement entre 2005 et 2015. Nous avons examiné leurs rapports pathologiques afin d'établir un diagnostic de GBM qui récidive ou les effets d'un traitement. Une analyse de la survie de ces patients a été ensuite menée en nous basant sur leurs taux de survie globale et leurs taux de survie sans aggravation (progression-free survival). À noter que nous avons également analysé les corrélations pouvant exister avec les séances de radiothérapie. Résultats : Au total, 123 patients ont été identifiés. De ce nombre, 116 avaient été soumis à une résection (94\%) alors que 7 avaient été soumis à une biopsie. Des changements à l'état de santé de ces patients en lien avec leurs traitements ont été signalés chez $16 \%$ d'entre eux $(\mathrm{n}=20)$. Ces derniers, soulignons-le, ont donné à voir des taux médians de survie globale et de survie sans progression plus élevés après une récidive que d'autres patients dont la progression de la maladie était réelle. Il convient toutefois de préciser qu'aucune différence notable n'a émergé en matière de taux de survie globale à partir d'un premier diagnostic. Mentionnons également que les effets des traitements sur les patients ont été associés à une intervention chirurgicale effectuée dans les 90 jours suivant la fin des séances de radiothérapie. Dans le cas de patients bénéficiant de telles séances au sein de notre établissement $(\mathrm{n}=53)$, un volume de cible de rayonnement plus important ainsi qu'une dose maximale plus élevée ont été associés aux effets évoqués ci-dessus. Conclusion : En somme, les effets de traitements ont été associés à la chirurgie au moment où l'on se rapprochait de la fin des séances de radiothérapie mais aussi à des volumes de cible de rayonnement plus importants ainsi qu'à des doses maximales

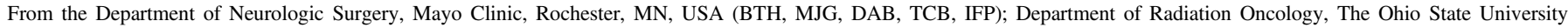

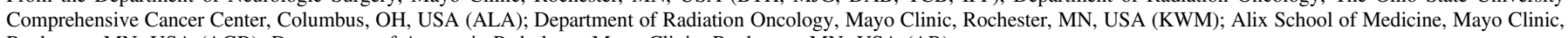
Rochester, MN, USA (AGB); Department of Anatomic Pathology, Mayo Clinic, Rochester, MN, USA (AR)

Received July 23, 2019. Final Revisions Submitted February 12, 2020. Date of Acceptance February 13, 2020.

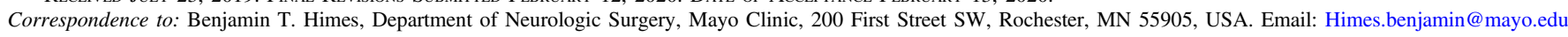


plus élevées. Ces mêmes effets de traitements ont été de surcroît associés à des taux de survie globale et de survie sans progression plus élevés à partir de la récidive des GBM et non pas à partir du moment où un premier diagnostic était posé.

Keywords: Glioblastoma, Pseudoprogression, Radiation necrosis

doi:10.1017/cjn.2020.36

Can J Neurol Sci. 2020; 47: 525-530

\section{INTRODUCTION}

Glioblastoma (GBM) is the most common primary malignant brain tumor in adults, and also carries the most dire prognosis, with a median survival of just over 14 months with current therapy. ${ }^{1,2}$ Treatment typically consists of maximal safe surgical resection, followed by fractionated radiation to a total dose of $60 \mathrm{~Gy}$ in 30 fractions with concomitant temozolomide. ${ }^{2}$ During treatment and thereafter, patients are followed closely with frequent magnetic resonance imaging (MRI) scans to assess for disease progression. Imaging evidence of recurrence typically dictates a change in therapy, whether with repeat surgery or additional drug therapy, such as with bevacizumab. ${ }^{1}$ However, treatment-related changes due to radiation and chemotherapy frequently mimic true disease progression, with increased T2 signal change and enhancement on MRI (Figure 1). This change can be transient and occurs early following the completion of therapy (so-called "pseudoprogression"), or take the form of delayed radiation necrosis that may occur many months after completion of radiation (these nonprogressive changes will be subsequently collectively referred to as "treatment effect" or "treatment-related changes"). ${ }^{3-5}$

A number of strategies have been put forth to evaluate MRI imaging to differentiate treatment effect from true disease progression. The MacDonald, Response Assessment in NeuroOncology (RANO) and Response Evaluation Criteria in Solid Tumors (RECIST) criteria have been employed with varying reported degrees of success. ${ }^{3,6,7}$ However, such systems are widely acknowledged to have significant flaws, and the need for improved diagnostic techniques and assessment of factors correlated with pseudoprogression remains critical. ${ }^{8}$ Of note, many series report rates of pseudoprogression or radiation necrosis diagnosis based purely on radiographic evidence, with patient survival frequently used as a retrospective surrogate for diagnosis in some series. ${ }^{9}$ This has led to some speculation that treatmentrelated changes may be correlated with longer overall survival (OS) in GBM patients - usually without supporting pathologic evidence. ${ }^{5,10}$ In contrast, others have reported that histopathology distinguishing recurrent tumor from radiation necrosis at resection for GBM recurrence has no prognostic value. ${ }^{11}$

In an effort to better characterize the clinical, radiographic, and treatment history of patients who demonstrate treatmentrelated changes during follow-up imaging, we sought to assess patients with pathology-proven evidence of treatment-related changes. To that end, we reviewed all patients at a single institution undergoing surgery (either biopsy or resection) for a presumed first recurrence of GBM.

\section{Methods}

Institutional Review Board (IRB) approval was obtained prior to initiation of the study (IRB \#15-009656). A departmentally

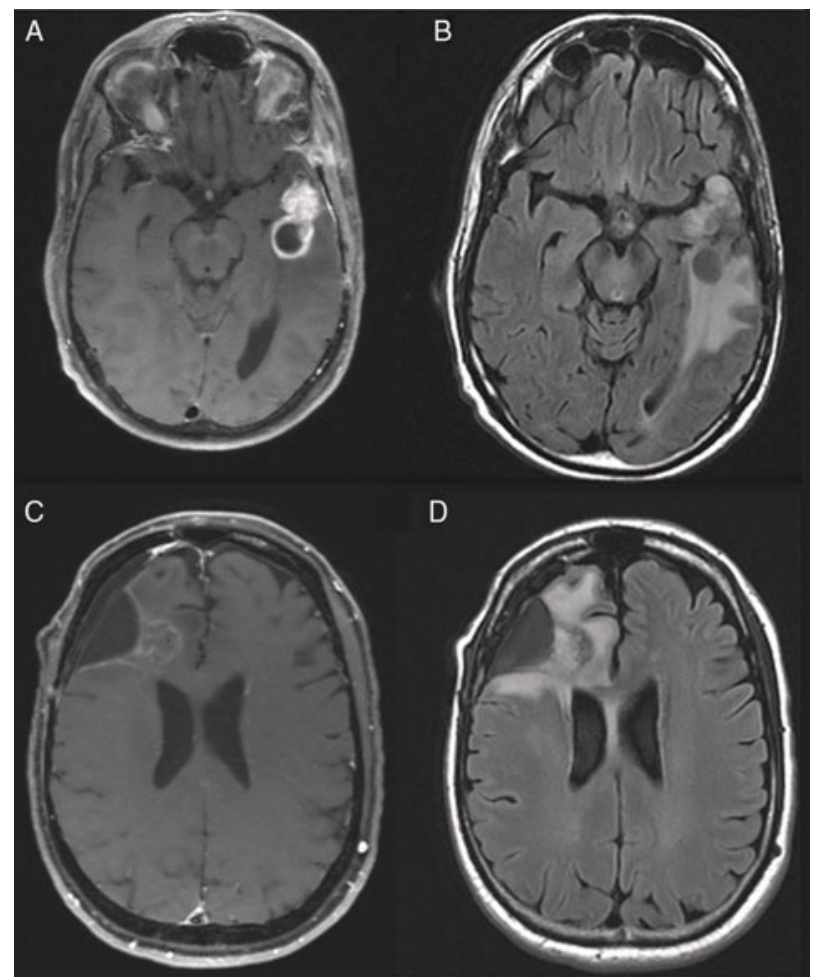

Figure 1: Representative images of treatment effect versus radiation necrosis in recurrent glioblastoma. (A and B) T1-weight post-contrast (A) and FLAIR $(B)$ images of an asymptomatic 57-year-old man with progressive disease 8 months after completion of radiation. ( $C$ and $D)$ $T 1$ with contrast $(C)$ and FLAIR $(D)$ images of a, asymptomatic 52-yearold man more than a year after completion of radiation; pathology demonstrated only treatment-related changes. Images are from patients included in this series.

maintained database of operative case logs at a single institution was reviewed for patients with a primary diagnosis of GBM who underwent surgical resection or biopsy for a presumed first recurrence between 2005 and 2015. Pathology records were reviewed to confirm a primary diagnosis of GBM and at recurrence for a diagnosis of recurrent GBM or treatment-related changes. In cases where the pathology report at recurrence was ambiguous, clinical records were reviewed to determine whether patients were treated as a true recurrence or pseudoprogression and categorized accordingly. All pathology reports were reviewed by a neuropathologist at our institution (AR) in order to ensure consistency in categorization, with this review providing definitive categorization. Clinical notes were reviewed to confirm tumor location, recurrence location, and clinical symptoms.

In cases where radiation plans were available (either for patients receiving radiation at our institution or for whom detailed 
Table 1: Demographic data

\begin{tabular}{|c|c|c|c|c|}
\hline & Overall & Progression & Treatment effect & $p$-value \\
\hline $\mathrm{N}$ & 123 & 103 & 20 & \\
\hline Median age (range) & $55(17-78)$ & $55(21-78)$ & $56(37-71)$ & 0.48 \\
\hline Female patients (\%) & $43(35)$ & $35(34)$ & $8(40)$ & 0.616 \\
\hline Biopsy (\%) & $7(6)$ & $4(4)$ & $3(16)$ & 0.068 \\
\hline Resection (\%) & $116(94)$ & $99(96)$ & $17(84)$ & 0.068 \\
\hline $\begin{array}{l}\text { Median time to first recurrence } \\
\text { (range in months) }\end{array}$ & $12.5(1.6-90.5)$ & $14.1(1.6-90.5)$ & $8.4(3.8-26.7)$ & 0.0003 \\
\hline Tumor location (\%) & & & & 0.695 \\
\hline Frontal & $36(29)$ & $28(27)$ & $8(40)$ & \\
\hline Temporal & $25(20)$ & $20(19)$ & $5(25)$ & \\
\hline Parietal & $20(16)$ & $17(17)$ & $3(15)$ & \\
\hline Occipital & $16(13)$ & $15(16)$ & $1(5)$ & \\
\hline Cerebellar & $2(2)$ & $2(2)$ & 0 & \\
\hline Multiple & $24(20)$ & $21(20)$ & $3(15)$ & \\
\hline
\end{tabular}

Bold indicates $p<0.05$

Table 2: Clinical characteristics associated with a diagnosis of treatment effect

\begin{tabular}{l|c|c|c}
\hline & Odds ratio & 95\% confidence & $\boldsymbol{p}$ \\
\hline interval & $0.90-21.27$ & 0.068 \\
\hline Preoperative steroid use & 4.37 & $1.08-14.16$ & $\mathbf{0 . 0 3 8}$ \\
\hline Recurrence out of field & 3.9 & $0.11-9.33$ & 0.98 \\
\hline Symptomatic recurrence & 1.03 & $0.51-3.61$ & 0.54 \\
\hline Recurrence <90 days after radiation & 1.36 & $1.99-34.22$ & $\mathbf{0 . 0 0 3 6}$ \\
\hline MGMT promoter methylation* & 8.25 & $0.19-2.74$ & 0.64 \\
\hline
\end{tabular}

Bold indicates $p<0.05$

*Results reported for 56 of 123 patients.

radiation planning records were imported (53 of 123 patients), plans were reviewed for volume treated, maximum total dose, maximum point dose, and volumes receiving $>105 \%$ of the prescribed dose. The type of radiation plan (3D conformal vs. intensity-modulated radiotherapy (IMRT)) was also recorded.

Statistical analysis was performed using GraphPad Prism8, Microsoft Excel 2010, and MedCalc. Kaplan-Meier curves were generated for comparing progression and OS rates, with surviving patients or those lost to follow-up censored, and time-to-event significance assessed via the Gehan-Breslow-Wilcoxon test, with $p$-values below 0.05 considered significant. Univariate analysis using odds ratios (ORs) was used to assess differences between treatment effect and true progression groups, with $p$-values below 0.05 reported as significant. Continuous variables reported as means were assessed via Student's $t$-test with $p$-values below 0.05 reported as significant.

\section{Results}

\section{Clinical Characteristics of Treatment-Related Changes}

Over a ten-year period (2005-2015) at a single institution, 123 cases of patients undergoing resection or biopsy for a presumed first recurrence of GBM were identified. Median age was 55 (21-78) in both groups and did not differ significantly between the two groups (age 55 in progression vs. 56 in treatment effect, $p=0.48$, Student's $t$-test). There were 35 female patients in the progression group $(35 \%)$ versus 8 patients in the treatmenteffect group ( $40 \%, p=0.616$, Fisher's Exact Test). One-hundredsixteen patients (94\%) underwent resection and $7(6 \%)$ underwent biopsy. Twenty patients $(16 \%)$ demonstrated treatment-related changes on final pathology (data summarized in Table 1).

Patients more commonly underwent biopsy at recurrence in the treatment-effect group ( $3 / 20$ cases, $15 \%)$ versus those with true disease progression (4/103 cases, $4 \%$ ); however, this trend did not reach statistical significance (OR 4.37, 95\% CI $0.89-21.28, p=0.068$, Table 2). A symptomatic presentation at recurrence was not associated with a specific diagnosis (OR 1.36, $95 \%$ CI $0.51-3.61, p=0.535$ ). However, preoperative steroid use (OR 3.9, 95\% CI 1.07-14.16, $p=0.0 .038$ ), or repeat surgery within 90 days following completion of radiation therapy, was associated with treatment-related changes on final pathology (OR 8.25, 95\% CI 1.99-34.22, $p=0.0036$ ). Overall time from diagnosis to first recurrence was also significantly shorter in the treatment-effect group (median survival 8.4 vs. 14.1 months, 


\section{Table 3: RANO criteria in treatment effect cases}

\begin{tabular}{l|c}
\hline & $\mathbf{N}(\%)$ \\
\hline$\geq 25 \%$ Increased enhancement & $11(52)$ \\
\hline Increased T2/FLAIR & $16(76)$ \\
\hline New lesion & $3(14)$ \\
\hline Clinical decline & $12(57)$ \\
\hline Cases meeting single criterion & $7(33)$ \\
\hline
\end{tabular}

$p=0.0003$, Gehan-Breslow-Wilcoxon test). Tumor location according to lobe (defined as either frontal, temporal, parietal, occipital, or multiple) was not associated with final pathologic diagnosis (Chi square 3.04, $p=0.695$ ). O6-methylguanine-DNA methyltransferase (MGMT) promotor methylation status was available in 56/123 cases (11/20 treatment-effect cases and 45/ 103 true progression cases). MGMT promotor methylation was not associated with a diagnosis of treatment-related changes (OR $0.73,95 \%$ CI $0.19-2.74, p=0.64)$.

The RANO criteria for progressive disease consist of any of the following: an at least $25 \%$ increase in the area of $\mathrm{T} 1$ gadolinium enhancement, significant new T2 or fluid-attenuated inversion recovery (FLAIR) signal, the presence of a new lesion, or clinical decline. Of the 20 patients with pathologic evidence of treatment effect, $100 \%$ met at least one criterion for progressive disease as follows: significant T2/FLAIR signal increase (15/20 cases), clinical decline (12/20 cases), and increased enhancement (11/20 cases). A single criterion was met in 7 of $20(35 \%)$ cases while no patient met all criteria. A new lesion was detected in 3 of 20 cases. These data are summarized in Table 3.

\section{Survival Analysis}

Patients with treatment-related changes on final pathology demonstrated prolonged median OS (from the time of surgery for presumed recurrence) relative to patients with true disease progression (16.5 vs. 10.3 months, $p=0.026,16$ of 132 cases censored due to ongoing survival or loss to follow-up Figure 2A). Median progression-free survival (PFS) was also longer in the treatment-effect group (6.5 vs. 4.3 months, $p=0.017$, 16 of 132 cases censored Figure 2B). However, when measured from the time of original disease diagnosis, statistical significance was lost, with median OS of 28.7 versus 28.1 months in the treatment effect versus true progression groups, respectively $(p=0.97$, 16 of 132 cases censored Figure 2C).

\section{Radiation Planning}

Fifty-three patients in the series underwent radiation therapy at our institution or had radiation planning records imported into our institution's planning software. The median radiation dose was $60 \mathrm{~Gy}$ in 30 fractions (range 39-76 Gy). The area of presumed recurrence occurred within the planned treatment volume (PTV) in all but two cases, one diagnosed as true progression and one as radiation necrosis. Detailed volumetric data were not able to be extracted in all cases, but where possible, we evaluated the conformity of the treatment plan.
The mean target volume receiving $105 \%$ of the prescribed dose (50.4cc vs. $16.2 \mathrm{cc}, p=0.013$ ) and the average maximum dose (69.9 vs. $64.2 \mathrm{~Gy}, p=0.008)$ were significantly higher in patients with treatment-related changes on final pathology (data available for $7 / 20$ treatment-effect cases for $105 \%$ of prescribed dose and 9/20 for maximum point dose, with data available for $39 / 103$ true progression cases in both cases). The volume of tissue receiving prescription dose was not associated with prediction of treatment effect (177cc vs. $193.4 \mathrm{cc}$ in cases of true progression, $p=0.689)$. Patients treated with IMRT had a higher rate of treatment-related changes than those treated with 3D conformal plans (OR 7.25, 95\% CI 1.32-39.5, $p=0.022$ ). However, these patients were more likely to have a volume of $105 \%$ prescription dose and a higher maximum point dose than patients receiving $3 \mathrm{D}$ conformal plans $(40.5 \mathrm{vs}$. $9.1 \mathrm{cc}, p=0.001$ and 68 vs. $63.4 \mathrm{~Gy}, p=0.007$, respectively). A number of patients with IMRT plans were also enrolled in dose-escalation studies where the total prescribed dose could be as high as 76 Gy. These data are summarized in Table 4.

\section{Discussion}

Here we describe a series of patients undergoing resection for a presumed first recurrence of GBM. We sought to assess the rate of pathology-proven treatment effect among patients that proceed with repeat surgery and to associate these findings with treatment-related variables. Many series on this subject report rates of pseudoprogression based solely on radiographic and clinical observation, rather than tissue diagnoses, which limits the assessment of patient's actual disease rate. ${ }^{9,12}$ In our series, the rate of treatment-related changes on final pathology was $16 \%$, similar to that reported in several other series. ${ }^{8,12,13}$ Prior studies have also reported a higher rate of treatment-related changes in cases close to the completion of radiation, with one study reporting over $50 \%$ of patients undergoing repeat surgery within six months of completion of radiation demonstrating radiation necrosis. ${ }^{13}$ The true rate of treatment-related changes masquerading as progression remains unclear; however, as most series on the subject are small and use varied criteria for defining treatmentrelated changes. ${ }^{14}$ Furthermore, many patients with new enhancement on MRI after radiation and temozolomide will not have surgery to obtain tissue prior to making treatment decisions. Therefore, we anticipate that the actual rate of treatment-related changes is likely substantially higher than commonly reported and likely higher than we report here, as we only identified patients who underwent a second surgery.

While many patients without pathologic evidence of disease progression were symptomatic on presentation (55\%) and may have benefited from a repeat surgical debulking regardless of the underlying pathology, our findings underscore the difficulty in distinguishing true progression from treatment effects based on radiographic and clinical criteria alone. Challenges in developing criteria for GBM progression based on imaging are widely reported, and multiple rubrics to assess disease progression have been put forth, including the MacDonald, RANO, and RECIST criteria. $^{3,6,7}$ Our hope in reviewing cases with pathologic diagnosis is to reduce ambiguity and potentially explore additional points of information that may provide valuable insight into the likelihood of pseudoprogression. 
Table 4: Radiotherapy planning factors*

\begin{tabular}{l|c|c|c}
\hline Plan type & 3D conformal & IMRT & $\boldsymbol{p}$ \\
\hline $105 \%$ Prescription dose volume (cc) & $9.1(20.8)$ & $40.5(41.7)$ & $\mathbf{0 . 0 0 1}$ \\
\hline Maximum point dose (Gy) & $63.4(0.14)$ & $68(0.84)$ & $\mathbf{0 . 0 0 8}$ \\
\hline & Progression & Treatment effect & $p$ \\
\hline 105\% Prescription dose volume (cc) & $16.2(27.5)$ & $50.4(52.3)$ & $\mathbf{0 . 0 1 3}$ \\
\hline Maximum point dose (Gy) & $64.2(0.31)$ & $69.9(11.4)$ & $\mathbf{0 . 0 0 8}$ \\
\hline Planning target volume (cc) & $193.5(112.4)$ & $177(100.8)$ & 0.689 \\
\hline
\end{tabular}

Bold indicates $p<0.05$

*Values reported as mean (standard deviation).
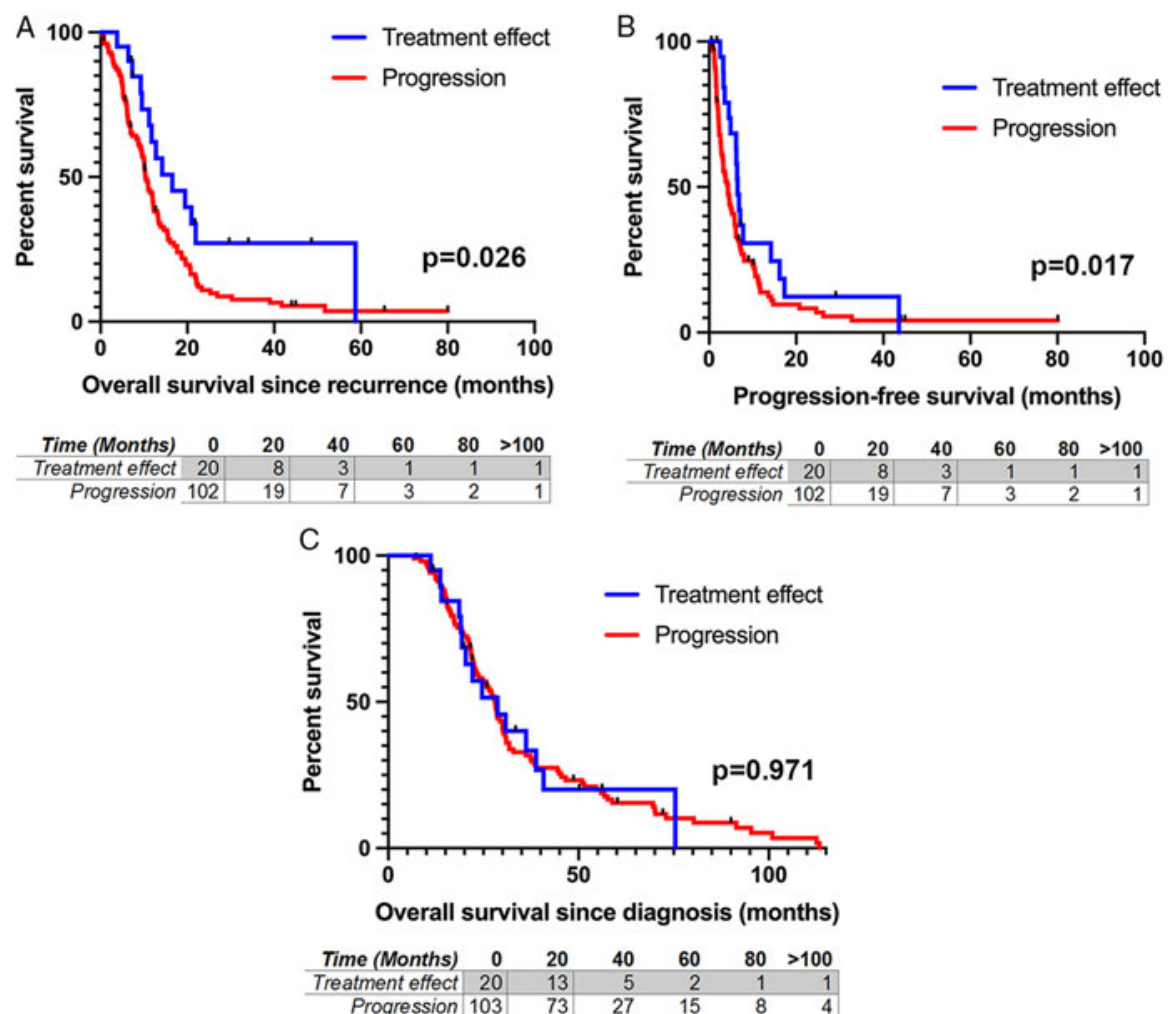

Figure 2: Survival analysis in recurrent glioblastoma. (A) Overall survival from the time of repeat resection for presumed GBM recurrence. (B) Progression-free survivalfrom the time of repeat resection as defined by subsequent radiographic progression. (C) Overall survival from time of initial GBM diagnosis. In all instances, blue lines represent patients with treatment-related changes on pathology, red lines represent true disease progression. Tables below each figure indicate the number of patients at risk at each given timepoint. p-values reported from Gehan-Breslow-Wilcoxon test.

To that end we closely reviewed available dose-volume histograms of radiation treatment plans for a subset of cases. We observed that heterogeneous plans with large volumes exceeding prescription dose were more likely to have treatmentrelated changes than plans that were more homogenous. The volume of tissue receiving doses greater than the prescription dose was a more important predictor of treatment effect than the total target volume receiving prescription dose. Further, only two patients were observed to have treatment-effect changes outside of the high dose target volume, indicating a dependency of radiation dose and volume on the development of pseudoprogression.
It has been suggested that pseudoprogression or treatmentrelated changes may correlate with increased survival in GBM. ${ }^{5,10,15}$ Indeed, when taken from the time of surgery for presumed recurrence, patients demonstrating treatment-related changes on pathology have longer overall and PFS times (Figure 2A and B). However, when the time of initial diagnosis of GBM is taken into account, this benefit vanishes, consistent with the finding that patients with pseudoprogression tend to have presumed recurrence closer to the completion of radiation, reported by others and confirmed in our series. ${ }^{13,15}$ That treatment effect does not appear to confer a survival benefit is important clinically, as this indicates that, where possible, 
measures should be taken to minimize the risk of treatmentrelated imaging changes in order to reduce the difficulty in assessing postoperative imaging, and in order to reduce the risk of unnecessary surgical interventions.

These findings underscore the need to delve further into the development of improved techniques for determining the likelihood of treatment-related changes. Efforts have been made to use MRI perfusion studies looking at increased relative cerebral blood volumes (rCBV) as a surrogate marker for disease progression with varying levels of efficacy. ${ }^{16-18}$ This technique was used intermittently in our series, but was not used as a major factor in determining whether patients had disease progression. Positronemission tomography (PET) studies have also been used to determine the likelihood of treatment effect, but significant logistical and cost constraints prevent this from being widely used. ${ }^{19}$

This study is limited by its relatively small size, the retrospective nature of the review, and by variability in the imaging modalities and criteria applied to each patient in the preoperative determination of likely disease progression. Further, a number of patients in this cohort remained censored in the survival analysis (16 total, 6 in the treatment-effect group with regard to OS since recurrence). The inability to follow all patients until mortality could potentially mask a survival benefit in the treatment-effect group. Additionally, while the express purpose of our study was to examine the rate of treatment-related changes in patients who proceed with repeat surgery, the generalizability to all patients with a primary diagnosis of GBM may be limited due to selection bias inherent in including patients that were healthy enough to undergo repeat surgery.

The need for improved characterization of postoperative imaging will only increase as the field moves forward, with the development of novel therapeutics including tumor vaccines and other immune-mediated approaches (several patients in our study ultimately received such therapies, but not prior to the first recurrence discussed here). Attempts have been made to take these treatments into account when assessing progression in the form of the iRANO criteria. ${ }^{20}$ Such treatments are frequently associated with significant radiographic treatment-related changes, which can make assessment of a patient's disease status incredibly difficult, especially in the context of assessing an investigational therapy, and ongoing efforts are necessary to better characterize this process and determine its implications for the clinical course of patients.

\section{CONFLict OF InTEREST}

The authors have no conflicts of interest to disclose.

\section{Statement of Authorship}

All authors contributed meaningfully to the development of this study and manuscript. Conception and design - BTH, AA, KWM, IFP. Data collection - BTH, AA, KWM, MG, AGB. Data analysis - BTH. Review of pathology findings - AR. Interpretation of data - all authors. Drafting and critical revision of manuscript - all authors.

\section{Ethical STandards}

IRB approval was received prior to initiation of the study (IRB $\# 15-009656$ ). This study is a retrospective review requiring review of the medical record only.

\section{REFERENCES}

1. Omuro A, DeAngelis LM. Glioblastoma and other malignant gliomas: a clinical review. JAMA. 2013;310:1842-50.

2. Stupp R, Mason WP, van den Bent MJ, et al. Radiotherapy plus concomitant and adjuvant temozolomide for glioblastoma. $\mathrm{N}$ Engl J Med. 2005;352:987-96.

3. Wen PY, Macdonald DR, Reardon DA, et al. Updated response assessment criteria for high-grade gliomas: response assessment in neuro-oncology working group. J Clin Oncol. 2010;28:1963-72.

4. Brandes AA, Tosoni A, Spagnolli F, et al. Disease progression or pseudoprogression after concomitant radiochemotherapy treatment: pitfalls in neurooncology. Neuro Oncol. 2008;10:361-7.

5. Hygino da Cruz LC Jr, Rodriguez I, Domingues RC, Gasparetto EL, Sorensen AG. Pseudoprogression and pseudoresponse: imaging challenges in the assessment of posttreatment glioma. AJNR Am J Neuroradiol. 2011;32:1978-85.

6. Eisenhauer EA, Therasse P, Bogaerts $\mathrm{J}$, et al. New response evaluation criteria in solid tumours: revised RECIST guideline (version 1.1). Eur J Cancer. 2009;45:228-47.

7. Macdonald DR, Cascino TL, Schold SC Jr, Cairncross JG. Response criteria for phase II studies of supratentorial malignant glioma. J Clin Oncol. 1990;8:1277-80.

8. Ellingson BM, Chung C, Pope WB, Boxerman JL, Kaufmann TJ. Pseudoprogression, radionecrosis, inflammation or true tumor progression? challenges associated with glioblastoma response assessment in an evolving therapeutic landscape. J Neurooncol. 2017;134:495-504.

9. Yun TJ, Park CK, Kim TM, et al. Glioblastoma treated with concurrent radiation therapy and temozolomide chemotherapy: differentiation of true progression from pseudoprogression with quantitative dynamic contrast-enhanced MR imaging. Radiology. 2015;274:830-40.

10. Brandsma D, Stalpers L, Taal W, Sminia P, van den Bent MJ. Clinical features, mechanisms, and management of pseudoprogression in malignant gliomas. Lancet Oncol. 2008;9:453-61.

11. Tihan T, Barletta J, Parney I, Lamborn K, Sneed PK, Chang S. Prognostic value of detecting recurrent glioblastoma multiforme in surgical specimens from patients after radiotherapy: should pathology evaluation alter treatment decisions? Hum Pathol. 2006;37:272-82.

12. Kucharczyk MJ, Parpia S, Whitton A, Greenspoon JN. Evaluation of pseudoprogression in patients with glioblastoma. Neuro-Oncol Pract. 2017;4:120-34.

13. Chamberlain MC, Glantz MJ, Chalmers L, Van Horn A, Sloan AE. Early necrosis following concurrent Temodar and radiotherapy in patients with glioblastoma. J Neurooncol. 2007;82:81-3.

14. Ellingson BM, Wen PY, van den Bent MJ, Cloughesy TF. Pros and cons of current brain tumor imaging. Neuro Oncol. 2014; 16(Suppl 7):vii2-11.

15. Sanghera P, Perry J, Sahgal A, et al. Pseudoprogression following chemoradiotherapy for glioblastoma multiforme. Can J Neurol Sci. 2010;37:36-42.

16. Kong D-S, Kim S, Kim E-H, et al. Diagnostic dilemma of pseudoprogression in the treatment of newly diagnosed glioblastomas: the role of assessing relative cerebral blood flow volume and oxygen6-methylguanine-DNA methyltransferase promoter methylation status. Am J Neuroradiol. 2011;32:382-87.

17. Gahramanov S, Muldoon LL, Varallyay CG, et al. Pseudoprogression of glioblastoma after chemo-and radiation therapy: diagnosis by using dynamic susceptibility-weighted contrast-enhanced perfusion MR imaging with ferumoxytol versus gadoteridol and correlation with survival. Radiology. 2013;266:842-52.

18. Young RJ, Gupta A, Shah AD, et al. MRI perfusion in determining pseudoprogression in patients with glioblastoma. Clin Imaging. 2013;37:41-9.

19. Galldiks N, Dunkl V, Stoffels G, et al. Diagnosis of pseudoprogression in patients with glioblastoma using O- $(2-[18 \mathrm{~F}]$ fluoroethyl $)-$ L-tyrosine PET. Eur J Nucl Med Mol Imaging. 2015;42: 685-95.

20. Okada H, Weller $M$, Huang $R$, et al. Immunotherapy response assessment in neuro-oncology: a report of the RANO working group. The Lancet Oncol. 2015;16:e534-42. 